\title{
Minimal Solutions for Generic Imaging Models
}

\author{
Srikumar Ramalingam ${ }^{1} \quad$ Peter Sturm $^{2}$ \\ ${ }^{1}$ Oxford Brookes University, UK \\ ${ }^{2}$ INRIA Grenoble Rhône-Alpes, France
}

\begin{abstract}
A generic imaging model refers to a non-parametric camera model where every camera is treated as a set of unconstrained projection rays. Calibration would simply be a method to map the projection rays to image pixels; such a mapping can be computed using plane based calibration grids. However, existing algorithms for generic calibration use more point correspondences than the theoretical minimum. It has been well-established that non-minimal solutions for calibration and structure-from-motion algorithms are generally noise-prone compared to minimal solutions. In this work we derive minimal solutions for generic calibration algorithms. Our algorithms for generally central cameras use 4 point correspondences in three calibration grids to compute the motion between the grids. Using simulations we show that our minimal solutions are more robust to noise compared to non-minimal solutions. We also show very accurate distortion correction results on fisheye images.
\end{abstract}

\section{Introduction}

Minimal solutions for computer vision algorithms have proven to be less noise-prone compared to non-minimal algorithms: they have been very useful in practice as hypothesis generators in hypothesize-and-test algorithms such as RANSAC [4]. Minimal solutions have been proposed for several computer vision problems: auto-calibration of radial distortion [12], perspective three point problem [5], the five point relative pose problem [17, 24, 15], the six point focal length problem $[25,13]$, the six point generalized camera problem [26], the nine-point problem for estimating paracatadioptric fundamental matrices [6] and the nine point radial distortion problem [14]. Inspired by these works, we add one more solution to the class of minimal solutions.

In our work we propose minimal solutions for the calibration of generic imaging models, which associate one projection ray to each individual pixel. By projection ray we refer to the 3D (half-) line along which light travels that falls onto the pixel (here, we neglect point spread and the finite spatial extent of a pixel). Rays may be unconstrained, i.e. they may not intersect in a single point, in which case the camera is called non-central. This general model has been used in various works $[8,16,18,19,20,22,23,29$, $30,31,32]$, and is best described in [8], where properties other than geometric ones are also considered. By adopting this model, one may formulate "black-box calibration" and provide algorithms that allow to calibrate any camera, be it of pinhole type (with or without optical distortions), catadioptric [1,11], pushbroom [9], or some other acquisi-

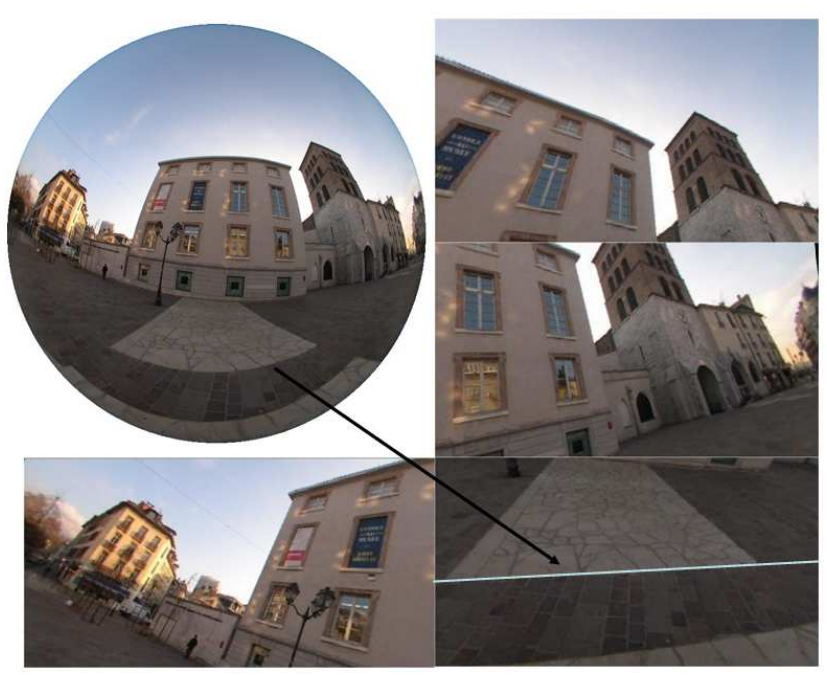

Figure 1. Distortion correction of a fisheye image after calibrating the camera using our 4-point algorithm. Note that the algorithm is capable of correcting highly distorted straight lines.

tion system [19, 23]. Such calibration algorithms have been proposed in $[2,7,8,29]$. A recent comparison paper shows that the generic calibration algorithms perform better than parametric approaches in the case of very high distortions [3].

In this paper, we adopt the approach of [29] which allows calibration from three or more images of a calibration grid, without having to know the motion between the images.

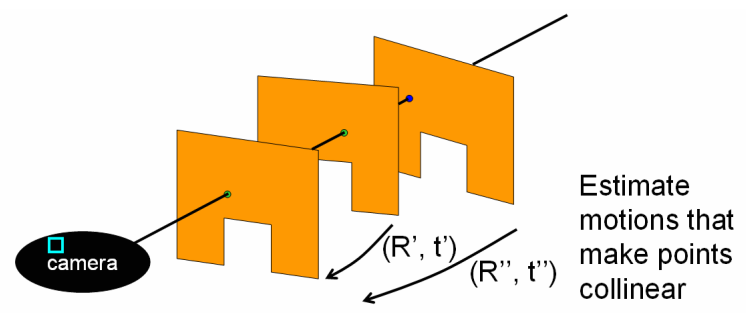

Figure 2. The concept of generic calibration. Three images of a calibration grid observed from different viewpoints and orientations are shown. A schematic diagram showing a single pixel and its $3 D$ ray. This ray is eventually computed by estimating the poses of the individual grids.

We briefly explain the main idea behind the generic calibration (see Figure 2). Three images of a calibration grid are captured from unknown viewpoints. Every image pixel observes three 3D points in different calibration grids. However, these points are collinear if they are expressed in the same coordinate system. This will enable us to compute the motion between the views and eventually the projection rays for the image pixels. 
It was shown in [29] that the generic calibration algorithm involves the computation of trifocal tensors. The motion parameters are not evaluated directly, rather they are first evaluated in coupled form and later extracted. One of the major drawbacks in generic calibration is that the current algorithm uses non-minimal approaches and the same motion variables can be computed from two different coupled variables. This leads to discrepancies in the computation of motion variables and some way of averaging should be used to obtain a consistent solution. Using minimum number of points, we avoid this problem and obtain a common consistent solution with respect to all the coupled variables. Later in section 4 we show that minimal solutions are more robust to noise compared to the non-minimal ones.

In this work we first present a minimal solution for the calibration of 2D central cameras. By 2D central cameras, we refer to cameras which live on a $2 \mathrm{D}$ plane and the projection rays intersect at a single point on the plane. This will best demonstrate our overall approach. Next we focus on the calibration of generally central cameras using three planar calibration grids. This is the most useful case in practice, as it models very accurately the commonly used cameras such as pinhole, fisheye and central catadioptric. We present a 4-point calibration algorithm for generally central cameras. As mentioned before, the calibration problem manifests itself as a motion estimation problem between the three calibration grids. Using triplets of 3D points, lying on the three calibration grids, for four pixels in the image, we compute the motion between the calibration grids. This will enable us to recover the projection rays. We recover all possible solutions, which is 8 in central models, and disambiguate using cheirality constraints. Our algorithm provides very accurate calibration results as shown in figure 1 . Note that our algorithm is capable of correcting highly curved straight lines.

Overview of the paper: Section 2 is intended to be a simple case emphasizing the main idea in our minimal solution algorithm. We present a generic calibration algorithm for a 2D central camera (all projection rays lie on a plane and intersect at a single point). In order to solve the calibration problem using minimal number of correspondences, we use orthogonality constraints and Gröbner basis computations. In section 3 we present a calibration algorithm for 3D central cameras (all projection rays intersect at a single point in space) using planar calibration algorithm. We use only 4 point correspondences in our algorithm, which involves simple algebraic transformations. Simulations are given to show that our minimal algorithms are more robust to noise compared to non-minimal ones in section 4 . We also present some real experiments for fisheye camera calibration using the 4-point algorithm. We conclude with a brief discussion about our work in section 5 .

\section{Central 2D Cameras}

Minimal case: We study the calibration problem on a 2D plane. The projection rays of the camera and the calibration grids, both lie on a plane. Let $\mathbf{Q}$ and $\mathbf{Q}^{\prime}$ refer to the known points on the first and the second calibration grids respectively. Let the coordinate system of the first calibration grid be the reference frame. Once the unknown motion between the two calibration grids $(R, t)$ is computed, projection rays can be recovered. Let $\mathbf{O}$ represent the unknown optical center. The number of independent degrees of freedom is 5 (1 for $\mathrm{R}^{\prime}$ and 2 for $\mathbf{t}^{\prime}, 2$ for $\mathbf{O}$ ).

We develop a counting argument for the minimal case. Since $\mathbf{O}, \mathbf{Q}$ and $\mathbf{Q}^{\prime}$ (after transforming to the reference frame) lie on a projection ray, we can impose collinearity constraint. Such a constraint is equivalent to a single equation in 2D space. This equation can be seen as the one that matches the slope computed from $\mathbf{O}$ and $\mathbf{Q}$ to the slope computed from $\mathbf{Q}$ and $\mathbf{Q}^{\prime}$. As a result, it can be easily seen that we need at least 5 pairs of grid points on 5 projection rays to solve the calibration problem. In the next section we present a calibration algorithm which uses 5 pairs of grid points in two calibration grids.

\subsection{Algorithm}

We use the constraint that with the correct estimates of the grid poses and the optical center, the latter is collinear with the two calibration points, for any pixel. The collinearity constraint will force the determinant of the following matrix to vanish:

$$
\begin{array}{ccc}
O_{1} & Q_{1} & R_{11}^{\prime} Q_{1}^{\prime}+R_{12}^{\prime} Q_{2}^{\prime}+t_{1}^{\prime} Q_{3}^{\prime} \\
O_{2} & Q_{2} & R_{21}^{\prime} Q_{1}^{\prime}+R_{22}^{\prime} Q_{2}^{\prime}+t_{2}^{\prime} Q_{3}^{\prime} \\
1 & Q_{3} & Q_{3}^{\prime}
\end{array} \mid=0
$$

\begin{tabular}{|r|r|r|}
\hline $\mathrm{i}$ & $C_{i}$ & $V_{i}$ \\
\hline 1 & $Q_{1} Q_{1}^{\prime}+Q_{2} Q_{2}^{\prime}$ & $R_{21}^{\prime}$ \\
\hline 2 & $Q_{1} Q_{2}^{\prime}-Q_{2} Q_{1}^{\prime}$ & $R_{22}^{\prime}$ \\
\hline 3 & $Q_{1} Q_{3}^{\prime}$ & $t_{2}^{\prime}-O_{2}$ \\
\hline 4 & $Q_{2} Q_{3}^{\prime}$ & $O_{1}-t_{1}^{\prime}$ \\
\hline 5 & $Q_{3} Q_{1}^{\prime}$ & $-O_{1} R_{21}^{\prime}+O_{2} R_{11}^{\prime}$ \\
\hline 6 & $Q_{3} Q_{2}^{\prime}$ & $-O_{1} R_{22}^{\prime}+O_{2} R_{12}^{\prime}$ \\
\hline 7 & $Q_{3} Q_{3}^{\prime}$ & $-O_{1} t_{2}^{\prime}+O_{2} t_{1}^{\prime}$ \\
\hline
\end{tabular}

Table 1. Coupled variables in the bifocal matching tensor for a central 2D camera.

This can be written as $\sum_{i=1} C_{i} V_{i}=0$, where $C_{i}$ and $V_{i}$ are given in Table 1. This is of the form $A_{n \times 7} \mathbf{V}_{7 \times 1}=0$. In order to solve the coupled variables $V_{i}$ 's uniquely we need at least 6 corresponding grid points. The existing generic calibration algorithm uses 6 point correspondences. We are interested in giving a minimal solution and we use only 5 pairs of grid points. Consequently we obtain the solution in a subspace spanned by two vectors. 


$$
\left(\begin{array}{c}
R_{21}^{\prime} \\
R_{22}^{\prime} \\
t_{2}^{\prime}-O_{2} \\
O_{1}-t_{1}^{\prime} \\
-O_{1} R_{21}^{\prime}+O_{2} R_{11}^{\prime} \\
-O_{1} R_{22}^{\prime}+O_{2} R_{12}^{\prime} \\
-O_{1} t_{2}^{\prime}+O_{2} t_{1}^{\prime}
\end{array}\right)=l_{1}\left(\begin{array}{c}
a_{1} \\
a_{2} \\
a_{3} \\
a_{4} \\
a_{5} \\
a_{6} \\
a_{7}
\end{array}\right)+l_{2}\left(\begin{array}{c}
b_{1} \\
b_{2} \\
b_{3} \\
b_{4} \\
b_{5} \\
b_{6} \\
b_{7}
\end{array}\right)
$$

The vectors $\mathbf{a}$ and $\mathbf{b}$ are known. There are 10 unknowns $\left(\mathrm{R}^{\prime}, \mathbf{O}, \mathbf{t}^{\prime}, l_{1}, l_{2}\right)$ and we have 7 equations. This implies that at least 3 more equations are needed to compute the unknowns. The remaining equations can be obtained from orthogonality constraints on the elements of $R^{\prime}$ :

$$
\begin{aligned}
R_{11}^{\prime} & =R_{22}^{\prime} \\
R_{21}^{\prime} & =-R_{12}^{\prime} \\
{R^{\prime 2}}_{21}^{2}+R_{22}^{\prime 2} & =1
\end{aligned}
$$

Using the above constraints along with equation (1), we express $R_{11}, R_{12}, R_{21}, R_{22}, O_{1}$ and $O_{2}$ as shown below:

$$
\begin{gathered}
\mathrm{R}^{\prime}=\left(\begin{array}{cc}
l_{1} a_{2}+l_{2} b_{2} & -l_{1} a_{1}-l_{2} b_{2} \\
l_{1} a_{1}+l_{2} b_{2} & l_{1} a_{2}+l_{2} b_{2}
\end{array}\right), \\
\mathbf{O}=\left(\begin{array}{c}
t_{1}^{\prime}+a_{4} l_{1}+b_{4} l_{2} \\
t_{2}^{\prime}-a_{3} l_{1}-b_{3} l_{2}
\end{array}\right)
\end{gathered}
$$

In what follows, we present the last three rows of equation (1) using the above equations:

$$
\begin{aligned}
-\left(t_{1}^{\prime}+a_{4} l_{1}+b_{4} l_{2}\right)\left(a_{1} l_{1}+b_{1} l_{2}\right)+\left(t_{2}^{\prime}+\right. & \\
\left.a_{3} l_{1}+b_{3} l_{2}\right)\left(a_{2} l_{1}+b_{2} l_{2}\right)-a_{5} l_{1}+b_{5} l_{2} & =0(7) \\
-\left(t_{1}^{\prime}+a_{4} l_{1}+b_{4} l_{2}\right)\left(a_{2} l_{1}+b_{2} l_{2}\right)-\left(t_{2}^{\prime}+\right. & \\
\left.a_{3} l_{1}+b_{3} l_{2}\right)\left(a_{1} l_{1}+b_{1} l_{2}\right)-a_{6} l_{1}+b_{6} l_{2} & =0(8) \\
-\left(t_{1}^{\prime}+a_{4} l_{1}+b_{4} l_{2}\right) t 2+\left(t_{2}^{\prime}+a_{3} l_{1}+b_{3} l_{2}\right) & \\
t_{1}^{\prime}-a_{7} l_{1}+b_{7} l_{2} & =0(9)
\end{aligned}
$$

There are three equations and four variables $l_{1}, l_{2}, t_{1}^{\prime}$ and $t_{2}^{\prime}$. The fourth constraint is obtained from equation 4 :

$$
\left(a_{1} l_{1}+b_{1} l_{2}\right)^{2}+\left(a_{2} l_{1}+b_{2} l_{2}\right)^{2}-1=0
$$

The above system has four equations(7-10) and four unknowns $l_{1}, l_{2}, t_{1}^{\prime}$ and $t_{2}^{\prime}$. We use Gröbner basis functions to solve the above system. The Gröbner basis for these four equations is given below:

$$
\begin{aligned}
& c_{11} l_{2}^{6}+c_{12} l_{2}^{4}+c_{13} l_{2}^{2}+c_{14}= 0 \\
& c_{21} l_{1}+c_{22} l_{2}^{5}+c_{23} l_{2}^{3}+c_{24} l_{2}=0 \\
& c_{31} t_{2}+c_{32} l_{2}^{5}+c_{33} l_{2}^{4}+c_{34} l_{2}^{3} \\
&+c_{35} l_{2}^{2}+c_{36} l_{2}+c_{37}= 0 \\
& c_{41} t_{1}+c_{42} l_{2}^{5}+c_{43} l_{2}^{4}+c_{44} l_{2}^{3} \\
&+c_{45} l_{2}^{2}+c_{46} l_{2}+c_{47}=0
\end{aligned}
$$

where $c_{i j}$ are known constraints that depend on $\boldsymbol{a}$ and $\boldsymbol{b}$. First we solve Equation 11 to obtain 6 different solutions for $l_{2}$. For each solution of $l_{2}$ we obtain solutions for $l_{1}$, $t_{2}$ and $t_{1}$. As a result, we obtain 6 different solutions for $\left(t_{1}^{\prime}, t_{2}^{\prime}, l_{1}, l_{2}\right)$. The equations 5 and 6 are used to compute $\mathrm{R}^{\prime}$ and $\mathbf{O}$.

Simulations were used to study the different solutions by randomly generating the rotation and translation variables. We conducted various tests. In one of them we obtained six solutions, where all of them were real ones. We show this test case in Figure 3. We use some kind of cheirality constraint to disambiguate the different solutions. As per this constraint, all calibration points associated to one pixel, should lie on the same half-line relative to the optical center. Note that the cheirality constraints that we are using here are different than the well-known ones for perspective cameras: for perspective cameras, the usual cheirality constraint means that points lie in front of the camera, a concept which is not directly applicable in omnidirectional cameras, especially for a field of view larger than $180^{\circ}$. Here, we only can impose that points seen by the same pixel lie on the same half-line relative to the optical center. In figure 3 we found that only one case satisfies the cheirality constraint. There were other cases where not all the six solutions were real. For instance, in one of the tests we obtained four imaginary solutions and two real ones. Only one of the two real solutions satisfied the cheirality constraint.

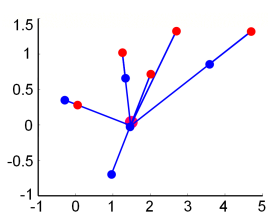

(a)

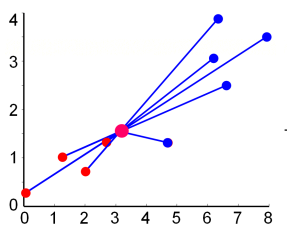

(d)

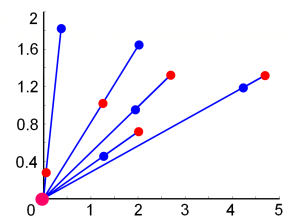

(b)

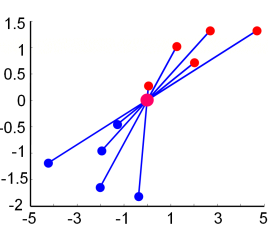

(e)

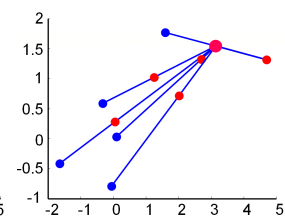

(c)
Figure 3. We show the calibration results. Points from a single calibration grid are represented by the same colour. Blue and Red color points refer to points on the first and the second calibration grids respectively. Six possible solutions are shown. The correct solution is the one shown in (b). In all other solutions the recovered points do not satisfy the cheirality constraint. (Best viewed in colour)

\section{Central 3D Cameras using planar grids}

Minimal Case: We consider the calibration problem of a central 3D camera using 3 planar calibration grids. First we 
address the question whether it is possible to use just two planar calibration grids to calibrate the camera. On examining the constraints, we found that they are insufficient to estimate the motion variables. This is to be expected since even for the pinhole model, full calibration using a planar calibration grid requires three views at least [27, 33].

Let us consider the coordinate system of the first calibration grid to be our reference coordinate system. Let $\left(\mathrm{R}^{\prime}, \mathrm{t}^{\prime}\right)$ be the motion between the first and the second grids. Let $\left(\mathrm{R}^{\prime \prime}, \mathrm{t}^{\prime \prime}\right)$ be the motion between the first and the second calibration grids. The total number of independent parameters to be computed is 15 ( 3 for $O, 6$ for $\left(R^{\prime}, t^{\prime}\right)$ and 6 for $\left.\left(\mathrm{R}^{\prime \prime}, \mathbf{t}^{\prime \prime}\right)\right)$.

Since we have 4 points $\left(\mathbf{O}, \mathbf{Q}, \mathbf{Q}^{\prime}\right.$ and $\left.\mathbf{Q}^{\prime \prime}\right)$ on every projection ray we have 2 collinearity constraints (collinearity constraint for $\left(\mathbf{O}, \mathbf{Q}, \mathbf{Q}^{\prime}\right)$ and $\left(\mathbf{O}, \mathbf{Q}, \mathbf{Q}^{\prime \prime}\right)$. Each collinearity constraint is equivalent to 2 equations in $3 \mathrm{D}$ space. Thus grid points on a single projection ray provides 4 equations. As a result we need at least 4 corresponding grid points to compute the 15 unknown parameters.

\subsection{Algorithm}

As explained in the $2 \mathrm{D}$ case the points are represented using their homogeneous coordinates (a $4 \times 1$ vector in $3 \mathrm{D}$ space). The coordinate system of the first calibration grid as the reference frame. We stack the three points $\mathrm{O}, \mathrm{Q}$ and $\mathrm{Q}^{\prime}$ (after transforming it to the reference frame:

$$
\left(\begin{array}{ccc}
O_{1} & Q_{1} & R_{11}^{\prime} Q_{1}^{\prime}+R_{12}^{\prime} Q_{2}^{\prime}+R_{13}^{\prime} Q_{3}^{\prime}+t_{1}^{\prime} Q_{4}^{\prime} \\
O_{2} & Q_{2} & R_{21}^{\prime} Q_{1}^{\prime}+R_{22}^{\prime} Q_{2}^{\prime}+R_{23}^{\prime} Q_{3}^{\prime}+t_{2}^{\prime} Q_{4}^{\prime} \\
O_{3} & Q_{3} & R_{31}^{\prime} Q_{1}^{\prime}+R_{32}^{\prime} Q_{2}^{\prime}+R_{33}^{\prime} Q_{3}^{\prime}+t_{3}^{\prime} Q_{4}^{\prime} \\
O_{4} & Q_{4} & t_{4}^{\prime} Q_{4}^{\prime}
\end{array}\right)
$$

As a consequence of the collinearity constraint the determinant of every $3 \times 3$ submatrix vanish. This results in equations of the form $\sum V_{i} Q_{j} Q_{k}^{\prime}=0$ involving bifocal tensors. Two of the four equations for collinearity constraint that uses $\mathbf{O}, \mathbf{Q}_{i}$ and $\mathbf{Q}_{i}^{\prime}$ are given in Table 2. In order to solve the above set of equations using least squares we need at least 5 triplets. However, we are interested in solving the minimal case by using only 4 triplets. As a result we will obtain the solution in a subspace spanned by two vectors as shown below:

$$
\left(\begin{array}{c}
R_{3,1}^{\prime} \\
R_{3,2}^{\prime} \\
-O_{3}+t_{3}^{\prime} \\
-O_{2} R_{3,1}^{\prime}+O_{3} R_{2,1}^{\prime} \\
-O_{2} R_{3,2}^{\prime}+O_{3} R_{2,2}^{\prime} \\
-O_{2} t_{3}^{\prime}+O_{3} t_{2}^{\prime}
\end{array}\right)=l_{1}\left(\begin{array}{c}
a_{1} \\
a_{2} \\
a_{3} \\
a_{4} \\
a_{5} \\
a_{6}
\end{array}\right)+l_{2}\left(\begin{array}{c}
b_{1} \\
b_{2} \\
b_{3} \\
b_{4} \\
b_{5} \\
b_{6}
\end{array}\right),
$$

$$
\left(\begin{array}{c}
R_{3,1}^{\prime} \\
R_{3,2}^{\prime} \\
-O_{3}+t_{3}^{\prime} \\
-O_{2} R_{3,1}^{\prime}+O_{3} R_{2,1}^{\prime} \\
-O_{2} R_{3,2}^{\prime}+O_{3} R_{2,2}^{\prime} \\
-O_{2} t_{3}^{\prime}+O_{3} t_{2}^{\prime}
\end{array}\right)=l_{3}\left(\begin{array}{c}
c_{1} \\
c_{2} \\
c_{3} \\
c_{4} \\
c_{5} \\
c_{6}
\end{array}\right)+l_{4}\left(\begin{array}{c}
d_{1} \\
d_{2} \\
d_{3} \\
d_{4} \\
d_{5} \\
d_{6}
\end{array}\right)
$$

The first three elements in Equations (16) and (17) are the same. As a result we get the following equations.

$$
\underbrace{\left(\begin{array}{llll}
a_{1} & b_{1} & -c_{1} & -d_{1} \\
a_{2} & b_{2} & -c_{2} & -d_{2} \\
a_{3} & b_{3} & -c_{3} & -d_{3}
\end{array}\right)}_{\mathrm{M}}\left(\begin{array}{c}
l_{1} \\
l_{2} \\
l_{3} \\
l_{4}
\end{array}\right)=0
$$

The rank of $M$ is 3. By solving the linear system, we will be able to compute $l_{1}, l_{2}, l_{3}$ and $l_{4}$ up to a scale. This implies that we will be able to express the coupled variables $V_{i}$ and $W_{i}$, given in Table 2 up to a scale. Let us assume that this common scale factor is $\lambda_{1}$. Using the same approach we also compute the coupled variables $M_{i}$ and $N_{i}$ up to a common scale $\lambda_{2}$.

$$
\left(\begin{array}{ccc}
H_{6 \times 2}^{\prime} & J_{6 \times 6} & 0_{6 \times 6} \\
H_{6 \times 2}^{\prime \prime} & 0_{6 \times 6} & J_{6 \times 6}
\end{array}\right)\left(\begin{array}{c}
-O_{1} \\
-O_{2} \\
X_{6 \times 1}^{\prime} \\
X_{6 \times 1}^{\prime \prime}
\end{array}\right)=\left(\begin{array}{c}
Y_{6 \times 1}^{\prime} \\
Y_{6 \times 1}^{\prime \prime}
\end{array}\right)
$$

$$
\begin{gathered}
H^{\prime}=\left(\begin{array}{ccc}
0 & l_{1} a_{1}+l_{2} b_{1} \\
0 & \begin{array}{l}
l_{1} a_{2}+l_{2} b_{2} \\
0
\end{array} \\
l_{1} a_{3}+l_{2} b_{3} \\
l_{1} a_{1}+l_{2} b_{1} \\
l_{1} a_{2}+l_{2} b_{2} \\
l_{1} a_{3}+l_{2} b_{3} \\
0 \\
0
\end{array}\right), Y^{i}=\left(\begin{array}{c}
l_{1} a_{4}+l_{2} b_{4} \\
l_{1} a_{5}+l_{2} b_{5} \\
l_{1} a_{6}+l_{2} b_{6} \\
l_{3} c_{4}+l_{4} d_{4} \\
l_{3} c_{5}+l_{4} d_{5} \\
l_{3} c_{6}+l_{4} d_{6}
\end{array}\right) \\
J=\left(\begin{array}{llllll}
0 & 0 & 0 & 0 & 1 & 0 \\
0 & 0 & 0 & 0 & 0 & 1 \\
0 & 1 & 0 & 0 & 0 & 0 \\
0 & 0 & 1 & 0 & 0 & 0 \\
0 & 0 & 0 & 1 & 0 & 0 \\
1 & 0 & 0 & 0 & 0 & 0
\end{array}\right), X^{\prime}=\left(\begin{array}{c}
\lambda_{i} O_{3}\left(t_{1}^{i}-O_{1}\right) \\
\lambda_{i} O_{3}\left(t_{2}^{i}-O_{2}\right) \\
\lambda_{i} O_{3} R_{1,1}^{\prime} \\
\lambda_{i} O_{3} R_{1,2}^{\prime} \\
\lambda_{i} O_{3} R_{2,1}^{\prime} \\
\lambda_{i} O_{3} R_{2,2}^{\prime}
\end{array}\right)
\end{gathered}
$$

The matrices $H^{\prime \prime}, X^{\prime}$ and $Y^{\prime \prime}$ are computed using the same approach. We rewrite equation (19) as follows:

$$
A_{12 \times(14)} u_{14}=Y_{14}
$$

Since $A$ is a matrix of rank 12 we express the 14 variables $u$ 's up to a linear combination of three vectors. Thus we have 15 motion parameters and 3 linear combination factors and thereby leading to 18 variables. We have only 14 equations that comes from the coupled variables. The method to compute the solution of equations of the form $A \mathbf{u}=\mathbf{b}$ using a linear combination of vectors including the null vectors is given in the appendix of [10]. We show the 14 elements of 


\begin{tabular}{|r|r|r|r|r|r|r|}
\hline $\mathrm{i}$ & $j$ & $k$ & $V_{i}$ & $W_{i}$ & $M_{i}$ & $N_{i}$ \\
\hline 1 & 1 & 1 & 0 & $R_{3,1}^{\prime}$ & 0 & $R_{3,1}^{\prime \prime}$ \\
\hline 2 & 1 & 2 & 0 & $R_{3,2}^{\prime}$ & 0 & $R_{3,1}^{\prime \prime}$ \\
\hline 3 & 1 & 4 & 0 & $-O_{3}+t_{3}^{\prime}$ & 0 & $-O_{3}+t_{3}^{\prime \prime}$ \\
\hline 4 & 2 & 1 & $R_{3,1}^{\prime}$ & 0 & $R_{3,1}^{\prime \prime}$ & 0 \\
\hline 5 & 2 & 2 & $R_{3,2}^{\prime}$ & 0 & $R_{3,2}^{\prime \prime}$ & 0 \\
\hline 6 & 2 & 4 & $-O_{3}+t_{3}^{\prime}$ & $-O_{3}+t_{3}^{\prime \prime}$ & $-O_{3}$ \\
\hline 7 & 4 & 1 & $-O_{2} R_{3,1}^{\prime}+O_{3} R_{2,1}^{\prime}$ & $-O_{1} R_{3,1}^{\prime}+O_{3} R_{1,1}^{\prime}$ & $-O_{2} R_{3,1}^{\prime \prime}+O_{3} R_{2,1}^{\prime \prime}$ & $-O_{1} R_{3,1}^{\prime \prime}+O_{3} R_{1,1}^{\prime \prime}$ \\
\hline 8 & 4 & 2 & $-O_{2} R_{3,2}^{\prime}+O_{3} R_{2,2}^{\prime}$ & $-O_{1} R_{3,2}^{\prime}+O_{3} R_{1,2}^{\prime}$ & $-O_{2} R_{3,2}^{\prime \prime}+O_{3} R_{2,2}^{\prime \prime}$ & $-O_{1} R_{3,2}^{\prime \prime}+O_{3} R_{1,2}^{\prime \prime}$ \\
\hline 9 & 4 & 4 & $-O_{2} t_{3}^{\prime}+O_{3} t_{2}^{\prime}$ & $-O_{1} t_{3}^{\prime}+O_{3} t_{1}^{\prime}$ & $-O_{2} t_{3}^{\prime \prime}+O_{3} t_{2}^{\prime \prime}$ & $-O_{1} t_{3}^{\prime \prime}+O_{3} t_{1}^{\prime \prime}$ \\
\hline
\end{tabular}

Table 2. Coupled variables in four of the bifocal matching tensors in a $3 \mathrm{D}$ single center camera with a planar calibration grid. Among the 16 tensors, there are 4 trifocal and 12 bifocal tensors.

u below:

$$
\left(\begin{array}{c}
O_{1} \\
O_{2} \\
\lambda_{1} O_{3}\left(t_{1}^{\prime}-O_{1}\right) \\
\lambda_{1} O_{3}\left(t_{2}^{\prime}-O_{2}\right) \\
\lambda_{1} O_{3} R_{1,1}^{\prime} \\
\lambda_{1} O_{3} R_{1,2}^{\prime} \\
\lambda_{1} O_{3} R_{2,1}^{\prime} \\
\lambda_{1} O_{3} R_{2,2}^{\prime} \\
\lambda_{2} O_{3}\left(t_{1}^{\prime \prime}-O_{1}\right) \\
\lambda_{2} O_{3}\left(t_{2}^{\prime \prime}-O_{2}\right) \\
\lambda_{2} O_{3} R_{1,1}^{\prime \prime} \\
\lambda_{2} O_{3} R_{1,2}^{\prime \prime} \\
\lambda_{2} O_{3} R_{2,1}^{\prime \prime} \\
\lambda_{2} O_{3} R_{2,2}^{\prime \prime}
\end{array}\right)=\left(\begin{array}{c}
a_{1} \\
a_{2} \\
a_{3} \\
a_{4} \\
a_{5} \\
a_{6} \\
a_{7} \\
a_{8} \\
a_{9} \\
a_{10} \\
a_{11} \\
a_{12} \\
a_{13} \\
a_{14}
\end{array}\right)+l_{1}\left(\begin{array}{c}
b_{1} \\
b_{2} \\
b_{3} \\
b_{4} \\
b_{5} \\
b_{6} \\
b_{7} \\
b_{8} \\
b_{9} \\
b_{10} \\
b_{11} \\
b_{12} \\
b_{13} \\
b_{14}
\end{array}\right)+l_{2}\left(\begin{array}{c}
c_{1} \\
c_{2} \\
c_{3} \\
c_{4} \\
c_{5} \\
c_{6} \\
c_{7} \\
c_{8} \\
c_{9} \\
c_{10} \\
c_{11} \\
c_{12} \\
c_{13} \\
c_{14}
\end{array}\right)
$$

Note that we have already used the vectors $\mathbf{a}, \mathbf{b}$ and $\mathbf{c}$ in equation (16). For simplicity we chose to use the same elements for subspace vectors in the above equation.

In order to compute $l_{1}$ and $l_{2}$ we use the constraints from the orthogonality properties of rotation matrix. We list the orthogonality constraints for $\mathrm{R}^{\prime}$ and $\mathrm{R}^{\prime \prime}$ :

$$
\begin{aligned}
& R_{11}^{\prime} R_{12}^{\prime}+R_{21}^{\prime} R_{22}^{\prime}+R_{31}^{\prime} R_{32}^{\prime}=0 \\
& R_{11}^{\prime \prime} R_{12}^{\prime \prime}+R_{21}^{\prime \prime} R_{22}^{\prime \prime}+R_{31}^{\prime \prime} R_{32}^{\prime \prime}=0 \\
& {R^{\prime}}_{11}^{2}+{R^{\prime 2}}_{21}^{2}+{R^{\prime 2}}_{31}^{2}=1 \\
& {R^{\prime 2}}_{12}^{2}+{R^{\prime 2}}_{22}^{2}+{R^{\prime 2}}_{32}^{2}=1 \\
& R^{\prime \prime 2}{ }_{11}^{2}+R_{21}^{\prime \prime 2}+R_{31}^{\prime \prime 2}=1 \\
& R_{12}^{\prime \prime^{2}}+R_{22}^{\prime{ }^{2}}+R_{32}^{\prime 2}=1
\end{aligned}
$$

Rewriting the above constraints using elements in equation (20):

$$
\begin{aligned}
u_{5} u_{6}+u_{7} u_{8}+V_{4} V_{5} O_{3}^{2} & =0 \\
u_{11} u_{12}+u_{13} u_{14}+M_{4} M_{5} O_{3}^{2} & =0 \\
u_{5}^{2}+u_{7}^{2}+\left(V_{4} O_{3}\right)^{2} & =\lambda_{1}^{2} O_{3}^{2} \\
u_{6}^{2}+u_{8}^{2}+\left(V_{5} O_{3}\right)^{2} & =\lambda_{1}^{2} O_{3}^{2} \\
u_{11}^{2}+u_{13}^{2}+\left(M_{4} O_{3}\right)^{2} & =\lambda_{2}^{2} O_{3}^{2} \\
u_{12}^{2}+u_{14}^{2}+\left(M_{5} O_{3}\right)^{2} & =\lambda_{2}^{2} O_{3}^{2}
\end{aligned}
$$

Note that $u_{i}$ are coupled variables of $l_{1}, l_{2}, \mathbf{a}, \mathbf{b}$ and c. We substitute for $u_{i}$ and rewrite the above equation to obtain the following linear system with eight variables: $l_{1}, l_{2}, l_{1} l_{2}, l_{1}^{2}, l_{2}^{2}, O_{3}^{2},\left(\lambda_{1} O_{3}\right)^{2}$ and $\left(\lambda_{2} O_{3}\right)^{2}$.

$$
\mathcal{A}_{6 \times 8}\left(\begin{array}{c}
l_{1} \\
l_{2} \\
l_{1} l_{2} \\
l_{1}^{2} \\
l_{2}{ }^{2} \\
O 3^{2} \\
\left(\lambda_{1} O 3\right)^{2} \\
\left(\lambda_{2} O 3\right)^{2}
\end{array}\right)=\left(\begin{array}{c}
a_{5} a_{6}+a_{7} a_{8} \\
a_{5}^{2}+a_{7}^{2} \\
a_{6}^{2}+a_{8}^{2} \\
a_{9} a_{10}+a_{11} a_{12} \\
a_{9}^{2}+a_{11}^{2} \\
a_{10}^{2}+a_{12}^{2}
\end{array}\right)
$$

where the eight columns of $\mathcal{A}$ are shown below.

$$
\begin{aligned}
& \mathcal{A}_{1}=\left(\begin{array}{c}
a_{5} b_{6}+b_{5} a_{6}+a_{7} b_{8}+b_{7} a_{8} \\
a_{11} b_{12}+b_{11} a_{12}+a_{13} b_{14}+b_{13} a_{14} \\
2 a_{5} b_{5}+2 a_{7} b_{7} \\
2 a_{6} b_{6}+2 a_{8} b_{8} \\
2 a_{11} b_{11}+2 a_{13} b_{13} \\
2 a_{12} b_{12}+2 a_{14} b_{14}
\end{array}\right), \\
& \mathcal{A}_{2}=\left(\begin{array}{c}
a_{5} c_{6}+c_{5} a_{6}+a_{7} c_{8}+c_{7} a_{8} \\
a_{11} c_{12}+c_{11} a_{12}+a_{13} c_{14}+c_{13} a_{14} \\
2 c_{5} a_{5}+2 c_{7} a_{7} \\
2 c_{6} a_{6}+2 c_{8} a_{8} \\
2 c_{11} a_{11}+2 c_{13} a_{13} \\
2 c_{12} a_{12}+2 c_{14} a_{14}
\end{array}\right), \\
& \mathcal{A}_{3}=\left(\begin{array}{c}
b_{5} c_{6}+c_{5} b_{6}+b_{7} c_{8}+c_{7} b_{8} \\
b_{11} c_{12}+c_{11} b_{12}+b_{13} c_{14}+c_{13} b_{14} \\
2 b_{5} c_{5}+2 c_{7} b_{7} \\
2 b_{6} c_{6}+2 c_{8} b_{8} \\
2 b_{11} c_{11}+2 c_{13} b_{13} \\
2 b_{12} c_{12}+2 c_{14} b_{14}
\end{array}\right)
\end{aligned}
$$




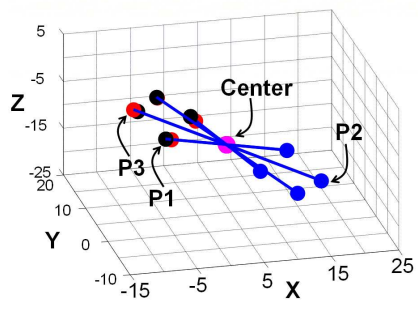

(a)

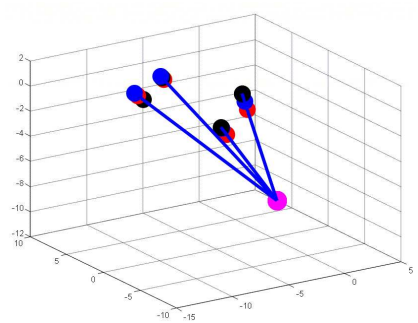

(e)

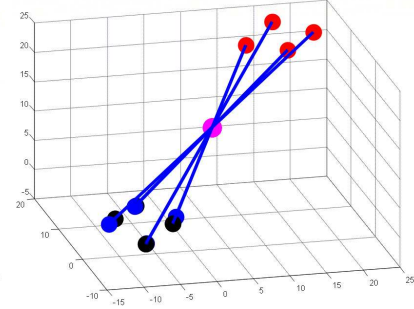

(b)

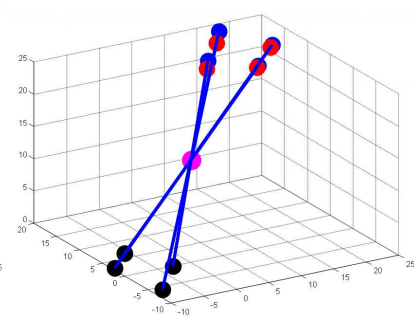

(f)

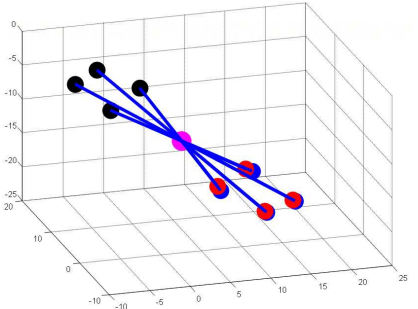

(c)

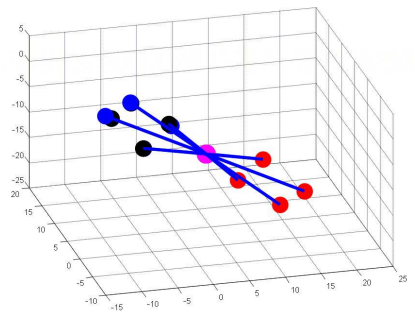

(g)

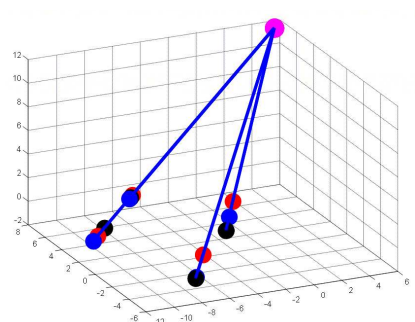

(d)

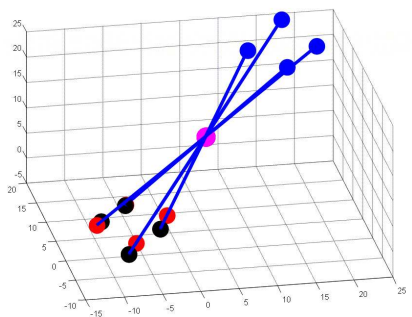

(h)

Figure 4. Calibration results for $3 D$ central camera. Points of the same colour belong to a specific calibration grid. Eight possible solutions are shown. Two of the solutions shown in $(d)$ and $(e)$ satisfy the cheirality constraint.

$\mathcal{A}_{4}=\left(\begin{array}{c}b_{5} b_{6}+b_{7} b_{8} \\ b_{11} b_{12}+b_{13} b_{14} \\ b_{5}{ }^{2}+b_{7}{ }^{2} \\ b_{6}{ }^{2}+b_{8}{ }^{2} \\ b_{11}{ }^{2}+b_{13}{ }^{2} \\ b_{12}{ }^{2}+b_{14}{ }^{2}\end{array}\right), \mathcal{A}_{5}=\left(\begin{array}{c}c_{5} c_{6}+c_{7} c_{8} \\ c_{11} c_{12}+c_{13} c_{14} \\ c_{5}{ }^{2}+c_{7}{ }^{2} \\ c_{6}{ }^{2}+c_{8}{ }^{2} \\ c_{11}{ }^{2}+c_{13}{ }^{2} \\ c_{12}{ }^{2}+c_{14}{ }^{2}\end{array}\right)$

$$
\mathcal{A}_{6}=\left(\begin{array}{c}
V_{4} V_{5} \\
M_{4} M_{5} \\
V_{4}^{2} \\
V_{5}^{2} \\
M_{4}^{2} \\
M_{5}^{2}
\end{array}\right), \mathcal{A}_{7}=\left(\begin{array}{c}
0 \\
0 \\
-1 \\
-1 \\
0 \\
0
\end{array}\right), \mathcal{A}_{8}=\left(\begin{array}{c}
0 \\
0 \\
0 \\
0 \\
-1 \\
-1
\end{array}\right)
$$

As a result we have eight variables in 6 equations. The rank of this system is 5 and thus we obtain the solution for these 8 variables in the subspace spanned by three vectors and linear combination factors $m_{1}$ and $m_{2}$.

$$
\left(\begin{array}{c}
l_{1} \\
l_{2} \\
l_{1} l_{2} \\
l_{1}^{2} \\
l_{2}^{2} \\
O_{3}^{2} \\
\left(\lambda_{1} O_{3}\right)^{2} \\
\left(\lambda_{2} O_{3}\right)^{2}
\end{array}\right)=\left(\begin{array}{c}
d_{1} \\
d_{2} \\
d_{3} \\
d_{4} \\
d_{5} \\
d_{6} \\
d_{7} \\
d_{8}
\end{array}\right)+m_{1}\left(\begin{array}{c}
e_{1} \\
e_{2} \\
e_{3} \\
e_{4} \\
e_{5} \\
e_{6} \\
e_{7} \\
e_{8}
\end{array}\right)+m_{2}\left(\begin{array}{c}
f_{1} \\
f_{2} \\
f_{3} \\
f_{4} \\
f_{5} \\
f_{6} \\
f_{7} \\
f_{8}
\end{array}\right)
$$

There are five variables and 8 equations. We will have to solve the above non-linear system to obtain $l_{1}$ and $l_{2}$. Us- ing simulations we verified that $e_{1}, e_{2}, f_{1}$ and $f_{2}$ are always zeros. This implies that $l_{1}$ and $l_{2}$ can be obtained uniquely. Using this result we compute the camera center as given below:

$$
\mathbf{O}=\left(\begin{array}{c}
a_{1}+l_{1} b_{1}+l_{2} c_{1} \\
a+2+l_{1} b_{2}+l_{2} c_{2} \\
\pm \sqrt{\frac{u_{5} u_{6}+u_{7} u_{8}}{-V_{4} V_{5}}}
\end{array}\right)
$$

The computed $\mathbf{O}$ is used to determine the scale factors $\lambda_{1}$ and $\lambda_{2}$

$$
\lambda_{1}= \pm \frac{\sqrt{u_{5}^{2}+u_{7}^{2}+\left(V_{4} O_{3}\right)^{2}}}{O 3}, \lambda_{2}= \pm \frac{\sqrt{u_{11}^{2}+u_{13}^{2}+\left(M_{4} O_{3}\right)^{2}}}{O 3}
$$

Using $\lambda_{1}, \lambda_{2}$ and $\mathbf{O}$ we present the rotation and translation parameters:

$$
\begin{aligned}
& \mathrm{R}^{\prime}=\left(\begin{array}{ccc}
\left(\frac{u_{5}}{O_{3} \lambda_{1}}\right) & \left(\frac{u_{6}}{O_{3} \lambda_{1}}\right) & \left(\frac{u_{7}}{O_{3} \lambda_{1}} \frac{V_{5}}{\lambda_{1}}-\frac{u_{8}}{O_{3} \lambda_{1}} \frac{V_{4}}{\lambda_{1}}\right) \\
\left(\frac{u_{7}}{O_{3} \lambda_{1}}\right) & \left(\frac{u_{8}}{O_{3} \lambda_{1}}\right) & \left(\frac{u_{6}}{O_{3} V_{1}}{\frac{1}{\lambda_{1}}}_{1} \frac{u_{5}}{O_{3} \lambda_{1}} \frac{M_{5}}{\lambda_{2}}\right) \\
\left(\frac{V_{4}}{\lambda_{1}}\right) & \left(\frac{V_{5}}{\lambda_{1}}\right) & \left(\frac{u_{5}}{O_{3} \lambda_{1}} \frac{u_{8}}{O_{3} \lambda_{1}}-\frac{u_{7}}{O_{3} \lambda_{1}} \frac{u_{6}}{O_{3} \lambda_{1}}\right)
\end{array}\right) \\
& \mathrm{R}^{\prime \prime}=\left(\begin{array}{ccc}
\left(\frac{u_{11}}{O_{3} \lambda_{2}}\right) & \left(\frac{u_{12}}{O_{3} \lambda_{2}}\right) & \left(\frac{u_{13}}{O_{3} \lambda_{2}} \frac{M_{5}}{\lambda_{2}}-\frac{M_{4}}{\lambda_{2}} \frac{u_{14}}{O_{3} \lambda_{2}}\right) \\
\left(\frac{u_{13}}{O_{3} \lambda_{2}}\right) & \left(\frac{u_{14}}{O_{3} \lambda_{2}}\right) & \left(\frac{u_{12}}{O_{3} \lambda_{2}} \frac{M_{4}}{\lambda_{2}}-\frac{M_{5}}{\lambda_{2}} \frac{u_{11}}{O_{3} \lambda_{2}}\right) \\
\left(\frac{M M_{4}}{\lambda_{2}}\right) & \left(\frac{M 5}{\lambda_{2}}\right) & \left(\frac{u_{11}}{O_{3} \lambda_{2}} \frac{u_{14}}{O_{3} \lambda_{2}}-\frac{u_{13}}{O_{3} \lambda_{2}} \frac{u_{12}}{O_{3} \lambda_{2}}\right)
\end{array}\right) \\
& \mathbf{t}^{\prime}=\left(\begin{array}{c}
\left(\frac{u_{3}+O_{1} O_{2} \lambda_{1}}{O_{3} \lambda_{1}}\right) \\
\left(\frac{u_{4}+O_{2} O_{3} \lambda_{1}}{O_{3} \lambda_{1}}\right) \\
\frac{O_{3} t_{2}^{\prime}-V_{9}}{O_{2}},
\end{array}\right) \mathbf{t}^{\prime \prime}=\left(\begin{array}{c}
\frac{u_{9}+O_{1} O_{2} \lambda_{2}}{O_{3} \lambda_{2}} \\
\frac{u_{10}+O_{2} O_{3} \lambda_{2}}{O_{3} \lambda_{2}} \\
\frac{O_{3} t_{2}^{\prime \prime}-M_{9}}{O_{2}},
\end{array}\right)
\end{aligned}
$$

Sign ambiguities exist for $O_{3}, \lambda_{1}$ and $\lambda_{2}$. As a result we have 8 possible solutions for the motion parameters. In 
figure 4 we show the eight solutions for a test case. Note that only two of the solutions satisfy the cheirality constraint. In order to disambiguate the solutions we can impose the constraint that $O_{3}$ has to be positive. This ensures that the calibration grid is in front of the optical center.

\section{Experiments}

Simulations: In order to compare the minimal solutions to the non-minimal ones we used simulations. We generated about 1000 random rays and used 4-point and 5-point algorithm embedded in a RANSAC framework. We plot the average angular error in the recovered projection rays with respect to Gaussian noise in the image. The image is assumed to be of size $500 \times 500$. The comparison is shown in figure 5. Note that our 4-point algorithm clearly outperforms the non-minimal 5-algorithm in the presence of noise.

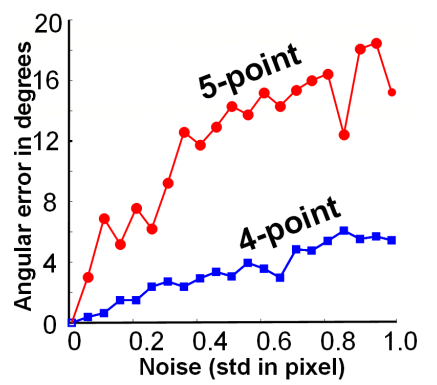

Figure 5. Comparison of 4-point and 5-point algorithms with respect to noise.

Fisheye Calibration: In order to test our calibration algorithm in practice we use Nikon Coolpix E8 fisheye cameras which has a very large field of view of $183^{\circ}$. We use white planar calibration grids with black dots on them as shown in Figure 6. For every image pixel we use interpolation to compute the 3D points in three different grids.

We use the a variant of the calibration algorithm using multiple grids proposed in [21]. The only difference being that we use our 4-point algorithm instead of their nonminimal 5-point algorithm. We used about 12 calibration grids to cover the whole fisheye image. The distortion correction is shown in figure 7 .

\section{Discussion}

This paper presents a minimal solution for the calibration of generally central camera model, which is the most useful one in practice. We have shown that the proposed algorithm is more robust to noise than the non-minimal one. We have shown promising result in distortion correction of fisheye lens. We are currently investigating minimal solutions for non-central camera models. In Table 3 we show the number of views and correspondences required for the algorithm given in [28].

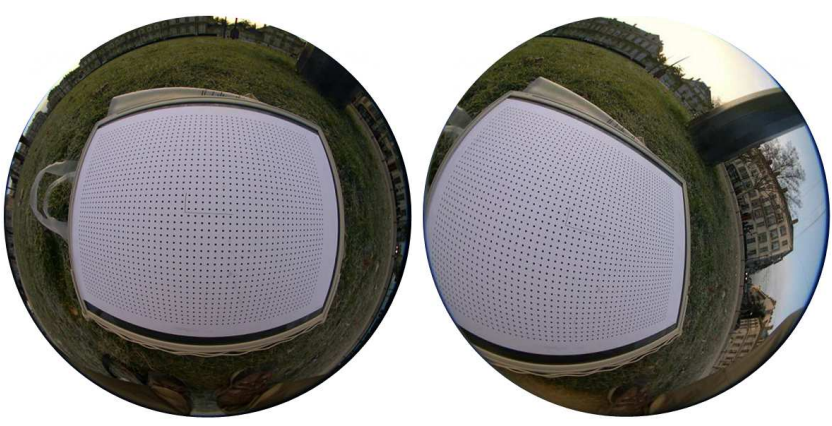

Figure 6. Two images of the grids used for calibrating the fisheye lens.

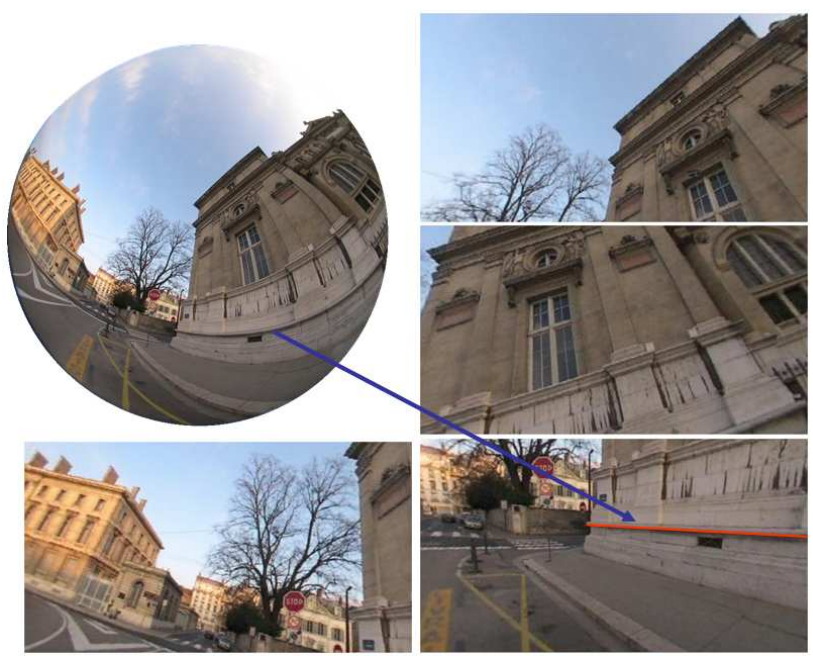

Figure 7. Distortion correction of a fisheye image using the calibration obtained using our 4-point algorithm.

\begin{tabular}{|c|c|c|}
\hline camera Model & {$[28]$} & Minimal Solution \\
\hline \hline $2 D$-C & $(2,6)$ & $(2,5)$ \\
\hline $3 D$-C & $(2,11)$ & $(2,5)$ \\
\hline $3 D$-C-Planar & $(3,5)$ & $(3,4)$ \\
\hline $2 D$-NC & $(3,12)$ & $(3,6)$ \\
\hline $3 D$-NC & $(3,29)$ & $(3,6)$ \\
\hline $3 D$ - $N$-Planar & $(3,18)$ & $(3,6)$ \\
\hline
\end{tabular}

Table $3.2 \mathrm{D}$ and $3 \mathrm{D}$ refer to $2 \mathrm{D}$ and $3 \mathrm{D}$ central cameras respectively. $\mathrm{C}$ and $\mathrm{NC}$ refer to central and non-central cameras respectively. Planar refers to planar calibration grids. $(n, m)$ refer to $n$ calibration grids using $m$ grid points in each of them.

Central 3D cameras using non-planar grids: We briefly give a counting argument for the minimal case for the calibration of central 3D camera using two 3D calibration grids. Let $\left(R^{\prime}, t^{\prime}\right)$ be the motion between the first and the second grids. Let $\mathbf{O}$ represent the optical center. The number of independent degrees of freedom is 9 ( 3 for $\mathbf{O}, 6$ for $\left(R^{\prime}, t^{\prime}\right)$ ). The points $\mathbf{O}, \mathbf{Q}$ and $\mathbf{Q}^{\prime}$ are collinear. Every collinearity constraint provides two equations in $3 \mathrm{D}$ space. We need at least 5 collinearity constraints to compute the 9 degrees of freedom.

For this case, we were successful in finding a calibration algorithm using 8 pairs of grid points (3 pairs more than the 
minimal case). Although this is much less than the number of points used by [29], the minimal case is 5 .

Non-central 3D cameras: Let $\left(R^{\prime}, t^{\prime}\right)$ and $\left(R^{\prime \prime}, t^{\prime \prime}\right)$ be the motion between the second and the third grids with respect to the reference frame (coordinate system of the first grid). The only available constraint is the collinearity constraint on every triplet of 3D points lying on the three grids corresponding to a specific pixel in the image. As seen earlier, this constraint is equivalent to two equations. In order to compute the 12 motion variables we need at least 6 point correspondences. In [29], the calibration was achieved using 29 point correspondences. This implies that, by using an approach similar to the one proposed in this paper, we will have to work in a subspace of 23 vectors.

The minimal cases of other scenarios, shown in Table 3 can be obtained in the same way. The remaining cases, other than the ones shown in this paper, are currently under investigation.

Acknowledgments Srikumar Ramalingam was supported by the EPSRC research grant EP/C006631/1(P). Peter Sturm was supported by the French ANR project CAVIAR.

\section{References}

[1] S. Baker and S. Nayar. A theory of catadioptric image formation. In ICCV, pages 35-42, 1998.

[2] G. Champleboux, S. Lavallée, P. Sautot, and P. Cinquin. Accurate calibration of cameras and range imaging sensors: the NPBS method. In ICRA, pages 1552-1558, 1992.

[3] A. Dunne, J. Mallon, and P. Whelan. A comparison of new generic camera calibration with the standard parametric approach. MVA, 2007.

[4] M. Fischler and R. Bolles. Random sample consensus: A paradigm for model fitting with applications to image analysis and automated cartography. Communications of the ACM, 1981.

[5] X. Gao, X. Hou, J. Tang, and H. Cheng. Complete solution classification for the perspective-three-point problem. 2003.

[6] C. Geyer and H. Stewenius. A nine-point algorithm for estimating para-catadioptric fundamental matrices. In $C V P R$, 2007.

[7] K. Gremban, C. Thorpe, and T. Kanade. Geometric camera calibration using systems of linear equations. In ICRA, pages 562-567, 1988.

[8] M. Grossberg and S. Nayar. A general imaging model and a method for finding its parameters. In ICCV, volume 2, pages 108-115, 2001.

[9] R. Gupta and R. Hartley. Linear pushbroom cameras. PAMI, 1997.

[10] R. Hartley and A. Zisserman. Multiple view geometry in computer vision. Cambridge University Press, 2000.

[11] R. Hicks and R. Bajcsy. Catadioptric sensors that approximate wide-angle perspective projections. In $C V P R$, pages 545-551, 2000.
[12] Z. Kukelova and T. Pajdla. A minimal solution to the autocalibration of radial distortion. In CVPR, 2007.

[13] H. Li. A simple solution to the six-point two-view focal length problem. In $E C C V, 2006$.

[14] H. Li and R. Hartley. A non-iterative method for correcting lens distortion from nine-point correspondenses. In $O M$ NIVIS, 2005.

[15] H. Li and R. Hartley. Five-point motion estimation made easy, 2006.

[16] J. Neumann, C. Fermüller, and Y. Aloimonos. Polydioptric camera design and $3 \mathrm{~d}$ motion estimation. In $C V P R$, volume 2, pages 294-301, 2003.

[17] D. Nistér. An efficient solution to the five-point relative pose problem. In $C V P R, 2003$.

[18] T. Pajdla. Stereo with oblique cameras. In $I J C V$, volume 47(1), 2002.

[19] S. Peleg, M. Ben-Ezra, and Y. Pritch. Omnistereo: Panoramic stereo imaging. In PAMI, pages 279-290, 2001.

[20] R. Pless. Using many cameras as one. In CVPR, pages 587594, 2003.

[21] S. Ramalingam, P. Sturm, and S. Lodha. Towards complete generic camera calibration. In $C V P R, 2005$.

[22] S. Seitz and J. Kim. The space of all stereo images. In International Journal of Computer Vision (IJCV), volume 48(1), pages 21-38, 2002.

[23] H. Shum, A. Kalai, and S. Seitz. Omnivergent stereo. In ICCV, pages 22-29, 1999.

[24] H. Stewenius, C. Engels, and D. Nister. Recent developments on direct relative orientation. ISPRS J. of Photogrammetry and Remote Sensing, 2006.

[25] H. Stewenius, D. Nister, F. Kahl, and F. Schaffalitzky. A minimal solution for relative pose with unknown focal length. In CVPR, 2005.

[26] H. Stewenius, D. Nister, M. Oskarsson, and K. Astrom. Solutions to minimal generalized relative pose problems. In OMNIVIS, 2005.

[27] P. Sturm and S. Maybank. On plane-based camera calibration. In $C V P R$, pages 432-437, 1999.

[28] P. Sturm and S. Ramalingam. A generic calibration concept: Theory and algorithms, research report 5058, inria. 2003.

[29] P. Sturm and S. Ramalingam. A generic concept for camera calibration. In $E C C V$, volume 2, pages 1-13, 2004.

[30] R. Swaminathan, M. Grossberg, and S. Nayar. A perspective on distortions. In CVPR, volume 2, page 594, 2003.

[31] Y. Wexler, A. Fitzgibbon, and A. Zisserman. Learning epipolar geometry from image sequences. In CVPR, 2003.

[32] D. Wood, A. Finkelstein, J. Hughes, C. Thayer, and D. Salesin. Multiperspective panoramas for cell animation. In SIGGRAPH, pages 243-250, 1997.

[33] Z. Zhang. A flexible new technique for camera calibration. PAMI, 2000. 